\title{
Effect of biosurfactant sophorolipids on Rhizomucor miehei lipase fermentation by Aspergillus oryzae
}

\author{
Qianqian Zhang ${ }^{1}$, Zhiyue Xiong ${ }^{1}$, Lei Sun ${ }^{1}$, Xiwei Tian' ${ }^{1}$, Guiwei Tian² ${ }^{2}$ Yiming Yang ${ }^{2}$, Xu Li ${ }^{*}$, \\ Yonghong Wang ${ }^{1}$ and Ju Chu ${ }^{1 *}$ (D)
}

\begin{abstract}
In this study, the effect of biosurfactant sophorolipids (SLs) on Rhizomucor miehei lipase (RML) fermentation by Aspergillus oryzae was investigated. With the exogenous addition of $0.3 \%(\mathrm{w} / \mathrm{v}) \mathrm{SLs}$ in the initial medium, the RML activity reached $430.0 \mathrm{U} / \mathrm{mL}$, an increase of $25.0 \%$ compared to the control group. Subsequently, the physiological metabolic responses of A. oryzae to the addition of SLs were further explored. The results showed that though SLs had almost no effect on the RML secretion, it would affect the morphology of the cells. During the late phase of the fermentation, the proportion of middle pellets, which was generally considered as an energetic and stable state for enzyme production was increased with the addition of SLS. Simultaneously, the viscosity of fermentation broth was reduced, which facilitated the increase of oxygen transfer, thereby improving the RML production. Finally, it could be found that the addition of SLs significantly increased the contents of precursor amino acids, especially for those rank first and second of the RML composition, and it could promote the synthesis of RML.
\end{abstract}

Keywords: Rhizomucor miehei lipase, Aspergillus oryzae, Sophorolipids, Morphology, Amino acid

\section{Introduction}

Rhizomucor miehei lipase (RML) is an $\alpha / \beta$-type singlechain polypeptide composed of 269 amino acids with a Ser-Asp-His catalytic triad structure (Boel et al. 1988; Huge-Jensen et al. 1989; Turkenburg et al. 1990). Due to its excellent interface activation, water-soluble RML can efficiently catalyze hydrolysis, transesterification, esterification and other reactions in non-aqueous reaction systems, so it has broad applications in oleochemistry, food processing, medicine, detergents and other industries (Sarmah et al. 2018; Torres and Otero 2001). However, the lipase production capacity of wild type $R$. miehei is relatively low, and the composition of the

\footnotetext{
*Correspondence: xuli@ecust.edu.cn; juchu@ecust.edu.cn

${ }^{1}$ State Key Laboratory of Bioreactor Engineering, East China University of Science and Technology, 130 Meilong Road, P.O. box 329, Shanghai 200237, People's Republic of China

Full list of author information is available at the end of the article
}

enzyme solution is unstable. Therefore, the RML production cost is pretty high, which greatly limits the industrial applications (Miao et al. 2013; Zhang et al. 2010). Researchers have been focusing on constructing heterologous expression systems for efficient RML production either by Aspergillus oryzae (Wang et al. 2009) or Pichia pastoris (Han et al. 2009; Liu et al. 2014).

Aspergillus oryzae, which is generally recognized as safe, is one of the important cell factories for protein production. However, compared with $P$. pastoris as the expression host of heterologous RML production, which could reach approximate $1200 \mathrm{U} / \mathrm{mL}$ (Huang et al. 2014), the expression level in $A$. oryzae seems to be much lower. Wang et al. (2009) successfully expressed RML in A. ory$z a e$ and obtained the integrated positive transformant $A$. oryzae ONL1, however, the enzyme activity of the fermentation supernatant measured by alkali titration was only $2.5 \mathrm{U} / \mathrm{mL}$ after 7 days cultivation. 
Surfactants are a class of organic compounds with hydrophilic and lipophilic properties. They have the functions of dispersion, emulsification, defoaming, penetration, solubilization, wetting, and adsorption. There have been many reports using surfactants as fermentation promoters for the production of amino acids (Huchenq et al. 1984), enzymes (Reddy et al. 1999) and new materials (Galindo and Salcedo 1996). Surfactants could improve the oxygen transfer at the gas-liquid interface and the permeability of cell membranes (Zhou 2012). Huchenq et al. (1984) studied the effect of surfactants on the fermentation of glutamate and found that surfactants have an inhibitory effect on the synthesis of fatty acids in the cell membrane, which resulted in changing the composition of the cell membrane and consequently increased the permeability to glutamate. As a kind of biosurfactant, SLs is a secondary metabolite produced by Candida sp. and has the advantages of low toxicity, biodegradability, high temperature and salt resistances ( $\mathrm{Li}$ et al. 2020). $\mathrm{Xu}$ et al. (2011) mentioned that the addition of SLs could induce the production efficiency of cellulase fermentation. And sophorose played a primary role as an effective inducer of cellulase synthesis.

In this study, the effects of five surfactants on the RML production by $A$. oryzae were investigated, and then the cellular metabolic responses to exogenous SLs addition for increasing RML activity were further explored from the perspectives of the broth rheological characteristics, the cell morphology and the cellular physiological metabolism.

\section{Materials and methods}

\section{Strain, media and cell cultivations}

Aspergillus oryzae was kindly provided by Wilmar Biotechnology R\&D Center Co., Ltd, Shanghai, China.

The seed medium $(\mathrm{g} / \mathrm{L})$ consisted of corn dextrin 20 , corn steep powder 10 , yeast extract powder 1 , polyether defoamer $1, \mathrm{KH}_{2} \mathrm{PO}_{4} 5, \mathrm{MgSO}_{4} \cdot 7 \mathrm{H}_{2} \mathrm{O} 0.5$, $\mathrm{Na}_{2} \mathrm{HPO}_{4} \cdot 12 \mathrm{H}_{2} \mathrm{O}$ 1. The composition of the fermentation medium ( $\mathrm{g} / \mathrm{L}$ ) was as follows: corn dextrin 40, peptone 30, yeast extract powder 4, polyether defoamer 1 , $\mathrm{KH}_{2} \mathrm{PO}_{4} 1.12, \mathrm{MgSO}_{4} \cdot 7 \mathrm{H}_{2} \mathrm{O} 0.5, \mathrm{Na}_{2} \mathrm{HPO}_{4} \cdot 12 \mathrm{H}_{2} \mathrm{O} 1$.

A. oryzae spore suspension was inoculated into 250$\mathrm{mL}$ shake flasks containing the seed medium. After incubating at $28{ }^{\circ} \mathrm{C}$ on a rotary shaker $(150 \mathrm{rpm})$ for $28 \mathrm{~h}$, the seed solution was transferred into $250-\mathrm{mL}$ shake flasks containing fermentation medium with $10 \%$ inoculum. The flasks were incubated at $28^{\circ} \mathrm{C}$ on a rotary shaker (200 rpm) for $168 \mathrm{~h}$.

\section{Screening of the surfactants and optimization of the SLs addition amount}

According to the addition amount of $0.2 \%(\mathrm{w} / \mathrm{v})$, five surfactants including Tween-80, acacia, polyvinyl alcohol, Triton X-100 and SLs (lactonic type, Lihan Biotech Co. Ltd, Shanghai, China) were added to the fermentation medium, respectively. Then, the flasks were incubated at $28^{\circ} \mathrm{C}$ on a rotary shaker $(200 \mathrm{rpm})$ for $168 \mathrm{~h}$. In detail, the SLs addition amount was set as $0.1 \%, 0.15 \%$, $0.2 \%, 0.25 \%, 0.3 \%, 0.35 \%$ and $0.5 \%$, respectively. Each set of experiments were repeated for three times.

\section{Extracellular RML enzyme activity assay}

RML activity in the diluted fermentation supernatant was determined by the $p$-nitrophenol ( $p$-NP) colorimetric method. One unit of RML activity was defined as the amount of enzyme, which liberated $1 \mu \mathrm{mol}$ of $p$ NP from $p$-nitrophenol palmitate ( $p$-NPP) per minute. The $p$-NPP solution and the substrate buffer containing phosphate buffer saline (PBS) were mixed in a ratio of $1: 9(\mathrm{v} / \mathrm{v}) .2 .4 \mathrm{~mL}$ of the mixed solution was pipetted into a $5 \mathrm{~mL}$ EP tube, and all tubes were preheated at $37^{\circ} \mathrm{C}$ for $3 \mathrm{~min}$. Next, $100 \mu \mathrm{L}$ of the diluted fermentation supernatant was added to the test tube, and $100 \mu \mathrm{L}$ of the boiled denatured sample solution was added to the control tube as a control. All the tubes were put in a constant temperature water bath oscillator at $37{ }^{\circ} \mathrm{C}$ for $15 \mathrm{~min}$. To stop the reaction, $2 \mathrm{~mL}$ of $95 \%$ ethanol was added immediately. Finally, all the tubes were centrifuged at $4000 \mathrm{rpm}$ for $5 \mathrm{~min}$. The absorbance of the sample and the control group were measured at $410 \mathrm{~nm}$. In addition, $p$-NP standard solutions of different concentrations were prepared and measured by same method. And a $p$-NP standard curve was acquired. RML enzyme activity $(\mathrm{U} / \mathrm{mL})$ was calculated according to formula (1):

$$
\text { Enzyme activity }=\frac{\left(A_{1}-A_{0}\right)+0.0015}{2.4684 * 15 * 0.1} * n,
$$

where $A_{1}$ is the absorbance of the sample, $A_{0}$ is the absorbance of the corresponding control group, $n$ is the dilution factor of the fermentation supernatant.

\section{Intracellular RML enzyme activity assay}

Pedersen's method was referred and modified slightly for intracellular RML enzyme activity assay (Pedersen et al. 1999). About $0.07 \mathrm{~g}$ of wet mycelia was weighted in a 2-mL EP tube, and $1 \mathrm{~mL}$ of $0.2 \mathrm{M} \mathrm{pH} 8.0 \mathrm{PBS}$ containing $1 \mathrm{mM}$ ethylene diamine tetraacetic acid (EDTA) was added to resuspend the mycelia. Then, $500 \mu \mathrm{L}$ of 
grinding beads ( $0.5 \mathrm{~mm}$ in diameter) were added in each EP tube, and all the tubes were put in a freezing grinder. The frequency and time were set as $65 \mathrm{~Hz}$ and $4 \mathrm{~min}$, respectively. Finally, all the samples were centrifuged at 12,000 rpm for $2 \mathrm{~min}$. The supernatant was acquired and the enzyme activity was determined by colorimetry.

Specifically, to acquire accurate intracellular activity proportion, the units of intracellular and extracellular enzyme activity had been normalized into units in the whole fermentation broth.

\section{Dry cell weight (DCW) assay}

The biomass of $A$. oryzae was indicated by the DCW. $20 \mathrm{~mL}$ of fermentation broth was filtered with a piece of dried filter paper, followed by repeated washing for 3 times with deionized water. And then the wet biomass was transferred to the electric oven at $80{ }^{\circ} \mathrm{C}$ for $24 \mathrm{~h}$ until the constant weight was obtained, after that the dried biomass was weighed immediately.

\section{Reducing sugar assay}

$1 \mathrm{~mL}$ of the diluted fermentation supernatant was put in a $10 \mathrm{~mL}$ colorimetric tube, then $1.5 \mathrm{~mL}$ of 3,5-dinitrosalicylic acid (DNS) reagent was added. After mixing well, all the colorimetric tubes were put in boiling water bath for $5 \mathrm{~min}$. When cooled to room temperature, all the reaction systems were diluted to $10 \mathrm{~mL}$. After mixing well, the absorbance of each sample was measured at $550 \mathrm{~nm}$. Glucose of different concentrations were prepared and measured by same method. And a glucose standard curve was acquired. Reducing sugar content $(\mathrm{g} / \mathrm{L})$ in the fermentation supernatant was calculated according to formula (2):

Reducing sugar content $=\left(1.3941 *\left(A_{1}-A_{0}\right)-0.018\right) * n$,

where $A_{1}$ is the absorbance of the sample, $A_{0}$ is the absorbance of the corresponding control group, $n$ is the dilution factor of the fermentation supernatant.

\section{Amino nitrogen assay}

$1 \mathrm{~mL}$ of fermentation supernatant was pipetted into a $150-\mathrm{mL}$ conical flask. First, $30 \mathrm{~mL}$ of distilled water and 2 drops of methyl red indicator were added and adjusted to light red with $0.1 \mathrm{M}$ hydrochloric acid, and left for $3 \mathrm{~min}$. Next, adjusted to light yellow with $0.05 \mathrm{M}$ sodium hydroxide solution. And then $5 \mathrm{~mL}$ of $18 \%$ neutral formaldehyde was added, mixed well and left for 3-5 min.
Last, 4 drops of phenolphthalein indicator were added and titrated with $0.05 \mathrm{M}$ sodium hydroxide solution to reddish color ( $\mathrm{pH} 8.0-10.0)$. The amino nitrogen content $(\mathrm{g} / \mathrm{L})$ in the fermentation supernatant was calculated according to formula (3):

$$
\text { Amino nitrogen }=\frac{c \times V_{1} \times 14}{V_{2}},
$$

where $c$ is the concentration of $\mathrm{NaOH}$ solution $(\mathrm{mol} / \mathrm{L})$, $V_{1}$ is the volume of $\mathrm{NaOH}$ solution $(\mathrm{mL})$ used for titration, $V_{2}$ is the volume of sample supernatant $(\mathrm{mL})$ used for determination.

\section{Broth viscosity assay}

About $15 \mathrm{~mL}$ of homogeneous fermentation broth was taken out, and then the viscosity was measured by LVDV$\mathrm{II}+\mathrm{P}$ viscometer with SC4-34 type rotor. And the specific parameters were as follows: the rotation speed was $200 \mathrm{rpm}$, and the temperature was room temperature.

\section{Determination of extracellular amino acids}

The S-433D amino acid analyzer (Jiesheng Yike Technology Development Co., Ltd, Beijing, China) was used to determine the extracellular amino acids concentrations in the fermentation supernatant. A total of $800 \mu \mathrm{L}$ of the fermentation supernatant was pipetted into a 1.5 -mL EP tube, and then $200 \mu \mathrm{L}$ of $10 \%$ sulfosalicylic acid was added. After mixing well, all the EP tubes were transferred at $4{ }^{\circ} \mathrm{C}$ for more than $30 \mathrm{~min}$, then they were centrifuged at 13,000 rpm for $10 \mathrm{~min}$. Next, the supernatant was diluted 3 times with lithium salt diluent, filtered through a $0.22-\mu \mathrm{m}$ filter membrane, and poured into a liquid phase vial for testing. After that, $100 \mu \mathrm{L}$ of the mixed amino acid standard and $900 \mu \mathrm{L}$ of the lithium salt diluent were mixed well, and poured into the liquid phase vial for testing. In addition, $1000 \mu \mathrm{L}$ lithium salt diluent was prepared as a blank. Finally, the free amino acid analysis standard program was called, the mixed amino acid standard was measured twice, then the lithium salt diluent was measured once, last the samples were measured.

\section{Determination of the pellet number and projected areas}

The quantitative analysis method of Aspergillus niger established by Tang et al. (2015) was referenced in this study. To fix pellets sample, $1 \mathrm{~mL}$ of fermentation broth was mixed with $1 \mathrm{~mL}$ of fixing agent consisting of $40 \%$ formaldehyde and $60 \%$ ethanol. After mixing, $300 \mu \mathrm{L}$ of the mixture was pipetted into a $5-\mathrm{mL}$ centrifuge tube, deionized water was added and mixed, then the centrifuge tube was kept stand for 5 min. Next, the upper 
suspended hyphae were wiped off, the bottom pellets were washed three times repeatedly with deionized water and diluted to $5 \mathrm{~mL}$ finally. Afterwards, the pellets sample was poured into a disposable plate of $8.5 \mathrm{~cm}$ and mixed with $20 \mathrm{~mL}$ of $60{ }^{\circ} \mathrm{C} 0.05 \%$ agarose. After cooling, each sample was photographed with a camera under black background. Last, Image J software was used to process the photos for parameter determination.

\section{Results and discussion}

\section{Selection of the optimal surfactant and its concentration}

Five surfactants, including Tween-80, acacia, polyvinyl alcohol, Triton X-100 and SLs, were tested in RML fermentation by A. oryzae. As shown in Fig. 1a, Tween-80, acacia, polyvinyl alcohol and Triton X-100 have strong inhibitory effect on the lipase production. The lowest enzyme activity dropped to about $70.0 \%$ of the control group by addition of acacia. In comparison, the addition of $0.2 \%$ SLs could significantly elevate the enzyme activity by $23.7 \%$. Zhou et al. (2012) reported that acacia and polyvinyl alcohol could promote $A$. oryzae CJLU-31 to produce lipase. Liu et al. (2012) found that both Triton X-100 and acacia could promote A. oryzae WZ007 to produce lipase, and $0.2 \%$ acacia exhibited the best effect. Differently, herein acacia, polyvinyl alcohol and Triton X-100 could not increase the RML activity, which might be due to that the mechanism of surfactants on the enzyme is closely relevant to the non-homologous structure of lipase, extracellular secretion, enzymatic properties and physiological metabolic characteristics of the host. Therefore, the same surfactant has different impact on different hosts and lipases.

Subsequently, the addition amount of SLs was further optimized. It could be found that when SLs concentration was in the range of $0.1 \%-0.5 \%$, it would not restrain A. oryzae from producing RML (Fig. 1b). When the addition amount of SLs was in the range of $0.1 \%-0.15 \%$, the increase in enzyme activity did not exceed $2.0 \%$ compared to the control group. Once the concentration increased to $0.3 \%$, the enzyme activity was significantly increased by $25.0 \%$, however, as the content of SLs further increased, the increase in enzyme activity showed a decreasing trend. Therefore, $0.3 \%$ addition of SLs was adopted in the following experiments.

\section{Effects of SLs on the intracellular and extracellular RML enzyme activities}

Although RML produced by $A$. oryzae is considered as an extracellular enzyme, it could also be detected intracellularly. The overall trends of the extracellular enzyme activity of both control group and experimental group (addition of $0.3 \%$ SLs) were described as follows. The extracellular enzyme activity increased over time during the whole fermentation process. When the cells entered the period
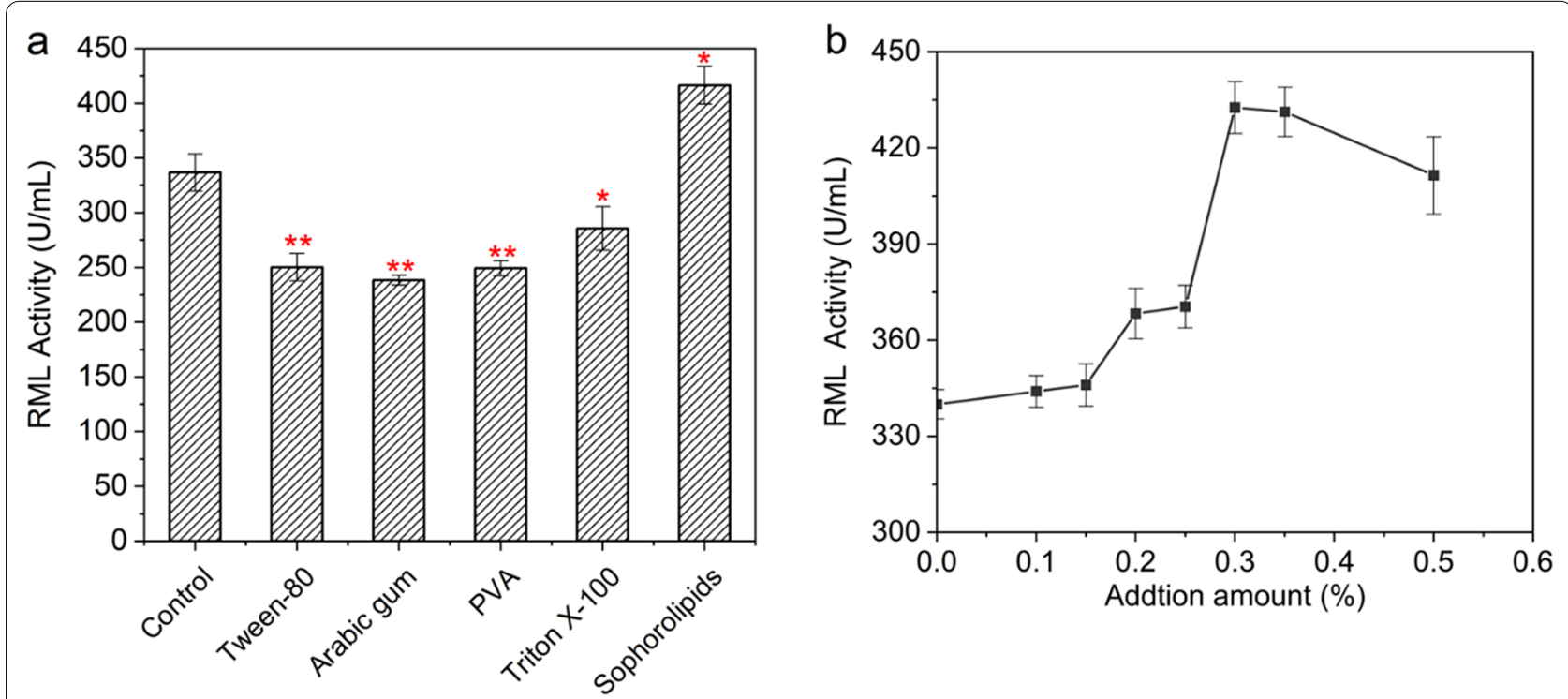

Fig. 1 Selection of the optimal surfactant and its concentration. a Effect of different surfactants on RML activity $\left({ }^{*}: 0.01<P \leq 0.05 ;{ }^{* *}\right.$ : P $\left.\leq 0.01\right)$. b Effect of different SLs concentrations on RML activity 

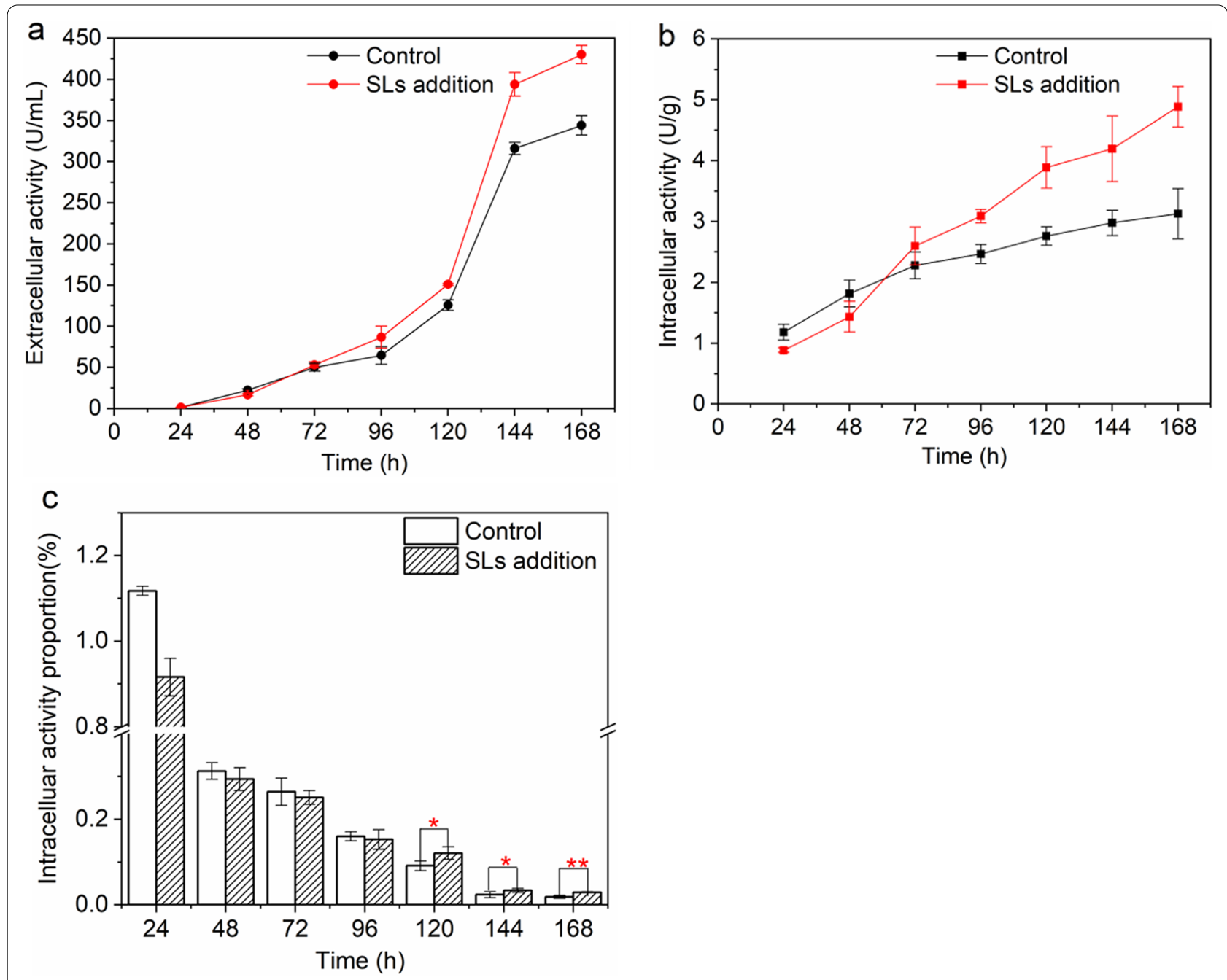

Fig. 2 Comparison of RML activities between control group and SLS addition group. a Extracellular activity. b Intracellular activity. c Intracellular activity proportion $(*: 0.01<P \leq 0.05 ; * *: \leq 0.01)$ When the intracellular activity proportion was calculated, the units of intracellular and extracellular enzyme activity had been normalized into units in the whole fermentation broth

of rapid enzyme production, especially after $120 \mathrm{~h}$, the increase rate of extracellular enzyme activity between SLs addition group and the control group was significantly different. At the end of fermentation (168 h), the extracellular enzyme activity of SLs addition group was 430.0 $\mathrm{U} / \mathrm{mL}$, which was $25.0 \%$ higher than that of the control group (Fig. 2a). Although the intracellular enzyme activity accounted for a very low proportion of the total enzyme activity (sum of intracellular and extracellular enzyme activity), it also could reflect the overall enzyme production. Obviously, starting from $48 \mathrm{~h}$, the increase rate of intracellular enzyme activity of SLs addition group exceeded that of the control group and lasted to the end of the fermentation (Fig. 2b). However, the highest intracellular enzyme activity accounted for only $1.1 \%$ of the total enzyme activity, indicating that the extracellular secretion of RML was relatively unhindered (Fig. 2c). Therefore, it was speculated that the addition of SLs would affect the cellular metabolism, and then promoted the RML synthesis, especially in the late stage (after $120 \mathrm{~h}$ ). These results seemed to be different from previous reports that the amphiphilic structure of surfactants might change the permeability of cell membranes and promote the secretion of extracellular enzymes (Zhou 2012). In the following experiments, the effects of 

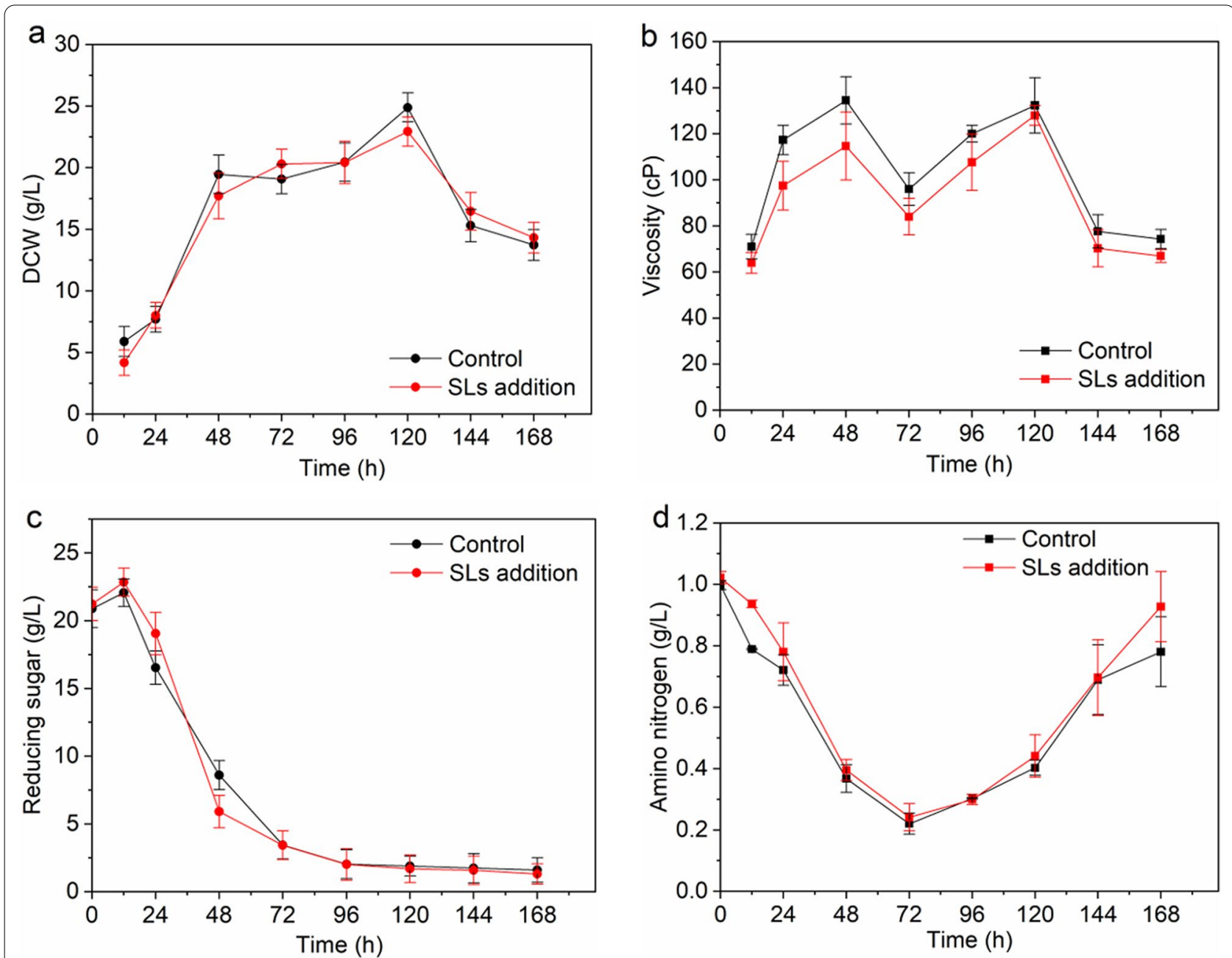

Fig. 3 Profiles of basic parameters during RML fermentation between control group and SLs addition group. a DCW; $\mathbf{b}$ viscosity; c reducing sugar; d amino nitrogen

SLs on the viscosity of the fermentation broth and cell morphology during the RML fermentation process were further explored.

\section{Effects of SLs on the broth rheological properties, cell morphology and cellular physiological metabolism}

The overall trends of the biomass characterized by DCW of the control group and SLs addition group during the RML fermentation process were relatively consistent (Fig. 3a). At 12 h, DCW in SLs addition group was $29.0 \%$ lower than that in the control group, which indicated that SLs would inhibit the cell growth to a certain extent. The rapid growth period of $A$. oryzae in both groups was 24-48 h. Correspondingly, at this period, reducing sugar and amino nitrogen were consumed fast (Fig. 3c and d). DCW decreased after $120 \mathrm{~h}$, which might be due to the autolysis of partially aged cells. During the fermentation process, the overall trend of the viscosity of the fermentation broth corresponded to the biomass (Fig. 3b). There was a phasic peak of viscosity at $48 \mathrm{~h}$ and $120 \mathrm{~h}$, respectively. Different from the trend of DCW, the viscosity decreased rapidly at $48-72 \mathrm{~h}$, which was mainly due to the significant change of morphology. Concretely, more dispersed hyphae aggregated into pellets, which greatly improved the rheological properties of the fermentation broth. It was worth noting that the viscosity of SLs addition group was lower than that of the control group during the whole fermentation process, especially during 

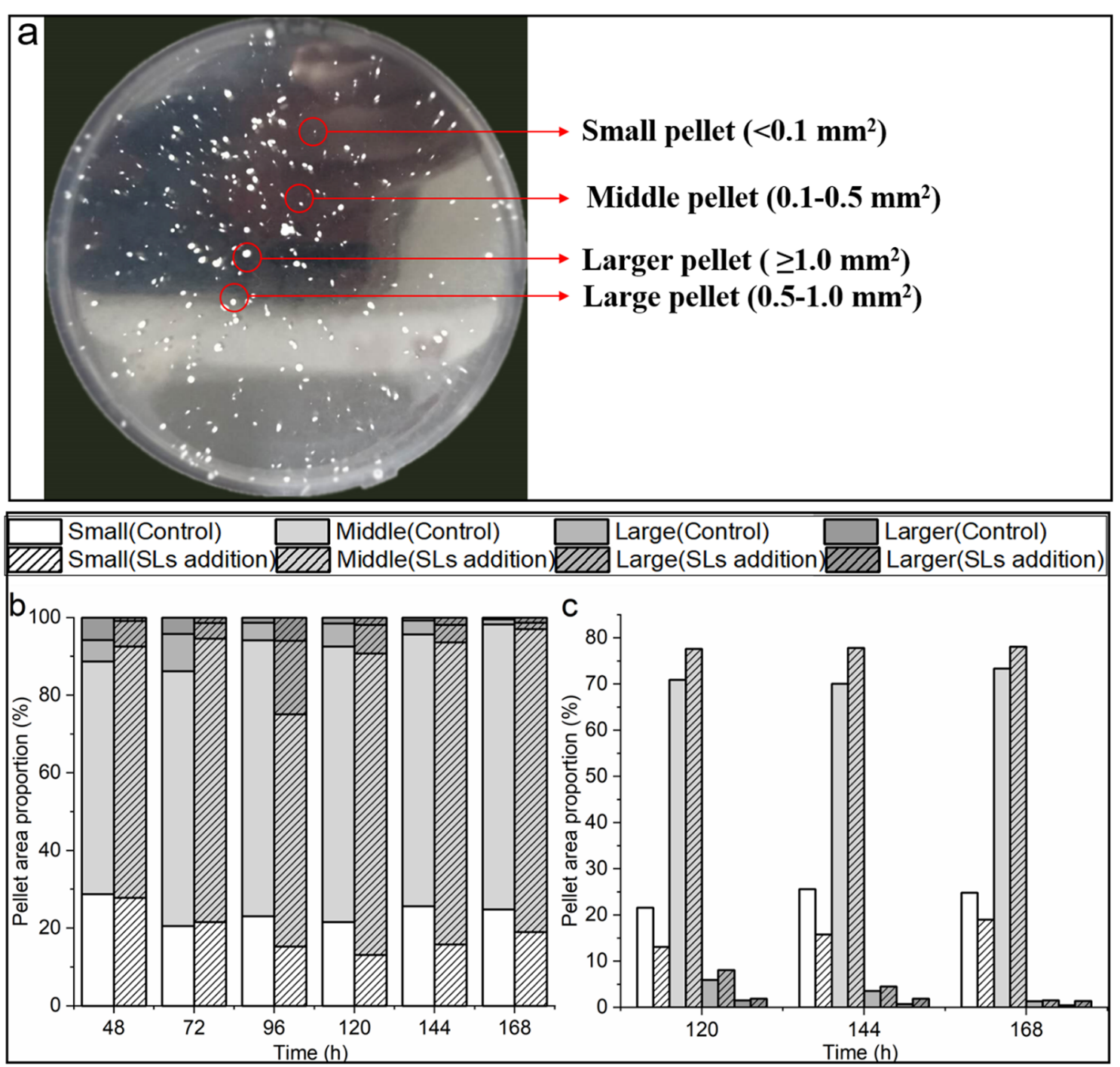

Fig. 4 Definition of four types of pellets and comparison between control group and SLS addition group. a The photo of A. oryzae pellets laid on an agarose plate. $\mathbf{b}$ Stacked column chart showing the proportion and their change of four kinds of pellets during the whole fermentation process. c The bar graph showing the difference of four kinds of pellets proportion between the control group and SLs addition group during the rapid enzyme production period

the early phase (24-72 h). This result indicated that SLs addition could reduce the viscosity of the fermentation broth, thereby improving the oxygen transfer in the environment. According to our previous work in a 50-L fermenter, the RML fermentation by $A$. oryzae is a highly aerobic process (data not shown), so starting from $48 \mathrm{~h}$, the RML production rate of SLs addition group exceeded that of the control group and maintained higher level.
The viscosity of the fermentation broth is not only related to biomass, but also closely related to morphology, and morphology is regulated by the cellular physiological metabolism and environmental parameters (Grimm et al. 2005; Riley et al. 2000). During RML fermentation process, $A$. oryzae exhibited different morphologies, ranging from dispersed mycelia to dense pellets, and simultaneously the sizes of pellets were 
different. Four types of pellets were defined according to the projection areas of pellets in this study (Fig. 4a). Small, middle, large and larger pellets refer to their projected areas $<0.1,0.1-0.5,0.5-1.0$ and $\geq 1.0 \mathrm{~mm}^{2}$, respectively. Both in the control and SLs addition groups, middle pellets accounted for the vast majority, followed by the small pellets during the whole fermentation process (Fig. 4b). In terms of small pellets, the number exhibited a trend of first decreasing and then increasing, which might be due to the gradual enlargement of small pellets in the first and middle stages of fermentation. However, when the fermentation reached $120 \mathrm{~h}$, the carbon source became insufficient, and the cells in the core parts of the large and larger pellets were aged, and then autolysis occurred which could provide nutrition and space for the growth of small pellets (White et al. 2002).

On the other hand, it was worth noting that compared to the control group, the proportion of small pellets was lower in SLs addition group, while the proportion of middle pellets was higher (Fig. 4c). These results indicated that the autolysis of the aging cells in SLs addition group provided more nutrients for small pellets to become middle pellets faster. And middle pellets were generally considered as an energetic and stable state for enzyme production. This might explain that the enzyme activity of SLs addition group was significantly higher than that of the control group during the period of rapid enzyme production. Chen et al. (2019) reported that the higher titer of L-malate by $A$. oryzae was achieved at a pellet number of $208 /$ $\mathrm{ml}$ and a pellet diameter of $1.05 \mathrm{~mm}$, not $1.12 \mathrm{~mm}$ or $1.02 \mathrm{~mm}$, indicating that middle pellets might have larger pellet surface for transport and nutrient uptake, and smoother surface for reducing viscosity. In addition, the cell morphology affects cellular physiological metabolism, and amino acids are necessary precursors for RML synthesis, so cellular amino acids metabolism with the SLs addition was analyzed subsequently.

The overall change trends of several extracellular amino acids concentrations were relatively consistent (Fig. 5). They were reduced to the minimum at $48 \mathrm{~h}$, indicating that the cells in rapid growth phase consumed a large amount of amino acids in the medium to synthesize mycelia protein and related enzymes. The rebound phenomenon began to appear at $120 \mathrm{~h}$, which may be due to the autolysis of aging cells. At the moment, protease activity increased, and more amino acids were released extracellularly, which was consistent with the rapid increase of the amino nitrogen content in the fermentation supernatant (Fig. 3d). Notably, after 120 h, most extracellular amino acids concentrations of SLs addition group were significantly higher than those of the control group, and the increase rates were also faster, especially serine, threonine, glutamate, aspartate and histidine. Among them, serine and threonine rank the first and second, respectively, in the amino acid composition of RML, while serine, histidine and aspartate are the components of the catalytic triad of RML. This phenomenon indicated that SLs addition group owned more precursor amino acids for RML synthesis. And this could also explain the RML enzyme activity and enzyme production rate of SLs addition group were significantly higher than those of the control group during the fast enzyme production period. Overall, SLs addition promoted the anabolism of RML precursor amino acids to a certain extent.

\section{Conclusions}

Exogenous addition of $0.3 \%(w / v)$ SLs could significantly enhance the RML production by $A$. oryzae. In detail, with the addition of SLs, the viscosity of fermentation broth during the fermentation process was significantly reduced, and the proportion of middle pellets, which was considered as energetic and stable state, was increased, thereby improving the RML production. Otherwise, an increase in precursor amino acids synthesis during the fast enzyme production period also contributed to the improved RML activity with the addition of SLs. This work was believed to be helpful to extend this strategy for further industrial application. 

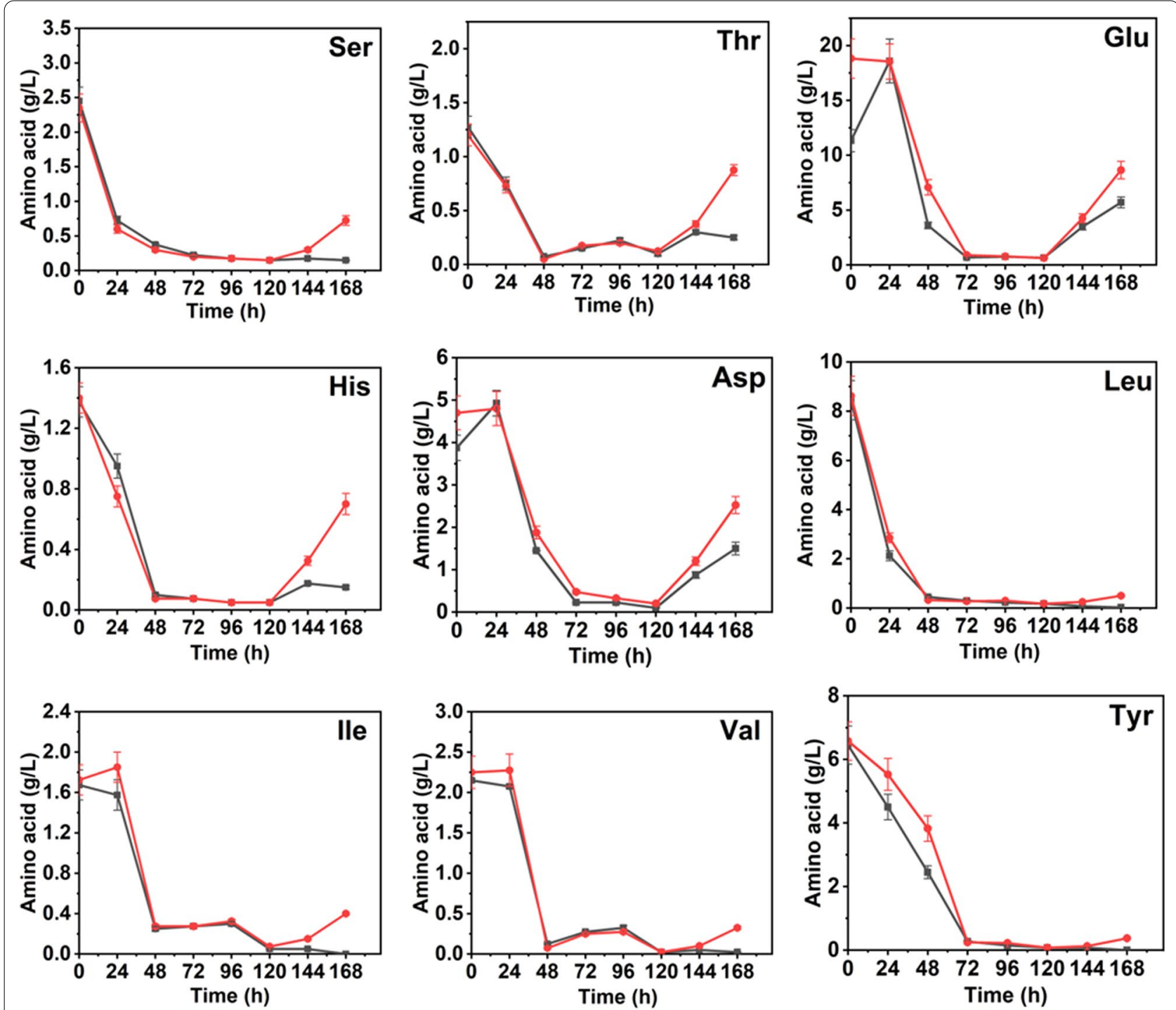

Fig. 5 Profiles of extracellular amino acids during RML fermentation between control group and SLs addition group. Nine representative amino acids including serine, threonine, glutamate, aspartate, histidine, leucine, isoleucine, valine and tyrosine were shown. The control groups were represented by black lines, and the SLs addition groups were represented by red lines

\section{Abbreviations}

RML: Rhizomucor miehei Lipase; A. oryzae: Aspergillus oryzae; p-NP: p-Nitrophenol; $p$-NPP: $p$-Nitrophenol palmitate; PBS: Phosphate buffer saline; EDTA: Ethylene diamine tetraacetic acid; DCW: Dry cell weight; SLs: Sophorolipids; Ser: Serine; Thr: Threonine; Glu: Glutamate; His: Histidine; Asp: Aspartate; Leu: Leucine; Ile: Isoleucine; Val: Valine; Tyr: Tyrosine.

\section{Acknowledgements}

We acknowledge Wilmar Biotechnology R\&D Center Co., Ltd for providing Aspergillus oryzae strain.

\section{Authors' contributions}

QZ, ZX and LS were in charge of the experiments and data analysis. GT and YY provided some guidance for material preparation and experimental operation. $\mathrm{XT}, \mathrm{XL}, \mathrm{YW}$ and JC provided valuable suggestions for experimental design and the organization of this manuscript. XT revised the manuscript draft written by QZ. All authors read and approved the final manuscript.
Funding

This work was financially supported by the National Key Research Development Program of China (2021YFC2100205, 2019YFA0904803) and the Fundamental Research Funds for the Central Universities, China (JKF01211708, JKVF1211036).

\section{Availability of data and materials}

The data supporting the conclusions of this article are included in the main manuscript.

\section{Declarations}

Ethics approval and consent to participate

Not applicable. 


\section{Consent for publication}

All of the authors have read and approved to submit it to Bioresources and Bioprocessing.

\section{Competing interests}

The authors declare that they have no competing interests.

\section{Author details}

${ }^{1}$ State Key Laboratory of Bioreactor Engineering, East China University of Science and Technology, 130 Meilong Road, P.O. box 329, Shanghai 200237 , People's Republic of China. ${ }^{2}$ Wilmar Biotechnology R\&D Center Co., Ltd, Shanghai 200137, China.

Received: 15 June 2021 Accepted: 18 August 2021

Published online: 03 September 2021

\section{References}

Boel E, Huge-Jensen B, Christensen M, Thim L, Fiil NP (1988) Rhizomucor miehei triglyceride lipase is synthesized as a precursor. Lipids 23(7):701-706

Chen X, Zhou J, Ding Q, Luo Q, Liu L (2019) Morphology engineering of Aspergillus oryzae for L-malate production. Biotechnol Bioeng 116(10):2662-2673

Galindo E, Salcedo G (1996) Detergents improve xanthan yield and polymer quality in cultures of Xanthomonas campestris. Enzyme Microb Tech 19(2):145-149

Grimm LH, Kelly S, Krull R, Hempel DC (2005) Morphology and productivity of filamentous fungi. Appl Microbiol Biot 69(4):375-384

Han Z, Han S, Zheng S, Lin Y (2009) Enhancing thermostability of a Rhizomucor miehei lipase by engineering a disulfide bond and displaying on the yeast cell surface. Appl Microbiol Biot 85(1):117-126

Huang J, Xia J, Yang Z, Guan F, Cui D, Guan G, Jiang W, Li Y (2014) Improved production of a recombinant Rhizomucor miehei lipase expressed in Pichia pastoris and its application for conversion of microalgae oil to biodiesel. Biotechnol Biofuels 7:111-121

Hucheng A, Marquet M, Welby M, Sheelu G (1984) Glutamate excretion triggering mechanism: a reinvestigation of the surfactant-induced modification of cell lipids. Ann Inst Pasteur Microbiol 135(1):53-67

Huge-Jensen B, Andreasen F, Christensen T, Christensen M, Thim L, Boel E (1989) Rhizomucor miehei triglyceride lipase is processed and secreted from transformed Aspergillus oryzae. Lipids 24(9):781-785

Li Y, Chen Y, Tian X, Chu J (2020) Advances in sophorolipid-producing strain performance improvement and fermentation optimization technology. Appl Microbiol Biot 104(24):10325-10337

Liu H (2012) Mutagenesis breeding of lipase producing Aspergillus oryzae WZ007 and separation purification of enzyme. Dissertation, Zhejiang University of Technology
Liu Y, Xie W, Yu H (2014) Enhanced activity of Rhizomucor miehei lipase by deglycosylation of its propeptide in Pichia pastoris. Curr Microbiol 68(2):186-191

Miao C, Luo W, Liu S, Liu P, Li H, Yang L, Yuan Z, Jiang J (2013) Cloning, expression and activity analysis of gene from Rhizomucor miehei lipase. Biotechnol Bull 6:128-133

Pedersen H, Carlsen M, Nielsen J (1999) Identification of enzymes and quantification of metabolic fluxes in the wild type and in a recombinant Aspergillus oryzae strain. Appl Environ Microb 65(1):11-19

Reddy RM, Reddy PG, Seenayya G (1999) Enhanced production of thermostable $\beta$-amylase and pullulanase in the presence of surfactants by Clostridium thermosulfurogenes SV2. Process Biochem 34(1):87-92

Riley GL, Tucker KG, Paul GC, Thomas CR (2000) Effect of biomass concentration and mycelial morphology on fermentation broth rheology. Biotechnol Bioeng 68(2):160-172

Sarmah N, Revathi D, Sheelu G, Yamuna Rani K, Sridhar S, Mehtab V, Sumana C (2018) Recent advances on sources and industrial applications of lipases. Biotechnol Progr 34(1):5-28

Tang W, Xia J, Chu J, Zhuang Y, Zhang S (2015) Establishment and application of the analysis method of cell morphology during the fermentation of Aspergillus niger. J Bioeng 31(2):291-299

Torres C, Otero C (2001) Direct enzymatic esterification of lactic acid with fatty acids. Enzyme Microb Tech 29(1):3-12

Turkenburg JP, Christiansen L, Huge-Jensen B, Norskov L, Brady L, Brzozowski AM, Derewenda ZS, Dodson E, Dodson G, Tolley S (1990) A serine protease triad forms the catalytic centre of a triacylglycerol lipase. Nature 343(6260):767-770

Wang B, Pan L, Guo Y (2009) Construction of heterogenous gene expression system in filamentous fungus Aspergillus oryzae. J South China Univ Technol 37(6):84-90

White S, Mclntyre M, Berry DR, McNeil B (2002) The autolysis of industrial filamentous fungi. Crit Rev Bitechnol 22(1):1-14

Xu Y, liu L, Li R, Guo H, Hu J, Han H, Wang X, Fan T (2011) Method for producing cellulase by inducing fermentation of sophorolipid. China Patent CN102250859A, 23 Nov 2011

Zhang Y, Zhao J, Yang B (2010) Cloning and expression of Rhizomucor miehei lipase and optimization of fermentation conditions. Anhui Agric Sci 35:19910-19913

Zhou J (2012) Screening and identification of a lipase producing strain, optimization of fermentation conditions and research on its enzymatic properties. Dissertation, China Institute of Metrology

\section{Publisher's Note}

Springer Nature remains neutral with regard to jurisdictional claims in published maps and institutional affiliations.

\section{Submit your manuscript to a SpringerOpen ${ }^{\circ}$ journal and benefit from:}

- Convenient online submission

- Rigorous peer review

- Open access: articles freely available online

- High visibility within the field

Retaining the copyright to your article

Submit your next manuscript at springeropen.com 